Case Report

\title{
Occult Bacteraemia and Aortic Graft Infection: A Wolf in Sheep's Clothing
}

\author{
E. Trautt, S. Thomas, J. Ghosh, P. Newton, and A. Cockcroft
}

University Hospital of South Manchester, Manchester, UK

Correspondence should be addressed to E. Trautt; elizabethtrautt@yahoo.co.uk

Received 30 April 2013; Accepted 10 July 2013

Academic Editors: R. A. Bishara, G. L. Tripepi, and R. Zbinden

Copyright (c) 2013 E. Trautt et al. This is an open access article distributed under the Creative Commons Attribution License, which permits unrestricted use, distribution, and reproduction in any medium, provided the original work is properly cited.

\begin{abstract}
We report a case of late-onset aortic prosthetic vascular graft infection. We stress the importance of maintaining a high index of suspicion for any patient presenting with fever on the background of in situ prosthetic material. We present the difficulties in managing these extremely complicated, often life and limb threatening infections and suggest that a multidisciplinary team approach, involving specialist centre referral, may be key to success. We highlight the difficulties in diagnosing late-onset PVGI, where presentation can be subacute with subtle signs and confusing microbiology. In this case the presentation was pyrexia of unknown origin with multiple positive blood cultures isolating a variety of gut-associated organisms; $a$ wolf in sheep's clothing.
\end{abstract}

\section{Background}

Prosthetic vascular graft infection (PVGI) is a significant complication of arterial reconstructive surgery [1]. The incidence has been reported to vary from 1 percent to 6 percent [2], depending on the site of the graft (infrainguinal 2-5\%, aortofemoral $1-2 \%$, and aortic $1 \%$ ). Although the relative risk of PVGI is low, the clinical consequences of an infected vascular graft can be catastrophic for the patient, with an associated operative morbidity of 40-70\% (limb amputation rates of up to $70 \%$ for lower extremity grafts [3]) and a recognized mortality rate of $30-50 \%$ (up to $75 \%$ with intra-abdominal aortic grafts [4]).

We describe an unusual presentation of late-onset aortic graft infection which stresses the importance of maintaining a high index of suspicion in any patient with unexplained fevers and underlying in situ prosthetic material. In management of this case we demonstrate effective clinical use of Daptomycin and highlight the need for national consensus guidelines to guide the management of these complex infections.

\section{Case Report}

This 79-years-old gentleman was admitted from the Infectious Diseases clinic, in April 2011. He presented to the clinic with night sweats, intermittent fevers, rigors, lethargy, weight loss, poor appetite, and generalised arthralgias. He described a change in the bowel habit over the previous few months with constipation and mild abdominal discomfort. His Creactive protein was 58 . This was his fourth follow-up clinic appointment following a recent hospital discharge.

His past medical history included the following: August 2010 admission for relapsed septic arthritis of a right native knee, joint fluid aspirated at that time isolated Pseudomonas aeruginosa and blood cultures repeatedly isolated Pseudomonas aeruginosa and Enterobacter; July 2010 admission for probable recurrent septic arthritis, blood cultures isolated Streptococcus constellatus and Aerococcus; December 2009 admission for a primary septic arthritis, culture of synovial fluid isolated Streptococcus constellatus. In 1993 he had undergone an aortic aneurysm repair with insertion of an aorto-biiliac Dacron surgical graft.

On his admission in December 2009, because of the in situ aortic graft, he had undergone a CT abdomen and pelvis which showed normal appearances of the aorto-bi-iliac graft. The scan was repeated on each subsequent admission, in July 2010 and August 2010, each time showing normal appearances of the graft and no evidence of a fluid or gas collection around the abdominal aorta or iliac arteries. On admission 
TABLE 1: Microbiology results.

\begin{tabular}{|c|c|c|c|c|}
\hline \multirow{2}{*}{ Date } & \multirow{2}{*}{ Clinical situation } & \multicolumn{2}{|c|}{ Microbiology } & \multirow{2}{*}{ Imaging } \\
\hline & & Sample site & Culture results & \\
\hline Dec 2009 & $\begin{array}{l}\text { Inpatient admission } \\
\text { Septic arthritis }\end{array}$ & Synovial fluid & $\begin{array}{l}\text { S. constellatus } \\
\text { (enrichment only) }\end{array}$ & $\begin{array}{l}\text { Jan. } 2010 \text { CT abdomen with contrast: no acute } \\
\text { abnormality detected }\end{array}$ \\
\hline Apr 2010 & $\begin{array}{l}\text { Inpatient admission } \\
\text { Relapsed septic arthritis }\end{array}$ & Blood & $\begin{array}{l}\text { S. constellatus } \\
\text { Aerococcus urinae }\end{array}$ & $\begin{array}{l}\text { Jul. } 2010 \text { CT abdomen and pelvis: no evidence of } \\
\text { graft infection }\end{array}$ \\
\hline Aug 2010 & $\begin{array}{l}\text { Inpatient admission } \\
\text { Recurrent septic arthritis }\end{array}$ & $\begin{array}{l}\text { Joint fluid } \\
\text { Blood (x3) }\end{array}$ & $\begin{array}{l}\text { Pseudomonas aeruginosa } \\
\text { Pseudomonas aeruginosa } \\
\text { Enterobacter cloacae }\end{array}$ & $\begin{array}{l}\text { Aug. } 2010 \text { CT angiogram aorta: no evidence of } \\
\text { abdominal aortic stent graft infection }\end{array}$ \\
\hline Mar 2011 & Outpatient clinic & Blood & Lactobacillus species & \\
\hline Apr 2011 & $\begin{array}{l}\text { Outpatient clinic } \\
\text { Admitted with PUO }\end{array}$ & Blood (x4) & $\begin{array}{l}\text { Lactobacillus paracasei } \\
\text { Lactobacillus species } \\
\text { S. oralis }\end{array}$ & $\begin{array}{l}\text { Apr. } 2011 \text { CT abdomen and pelvis: at least } \\
2 \text { small droplets of retroperitoneal gas which } \\
\text { appear extraluminal and suspicious for } \\
\text { sepsis related to the graft }\end{array}$ \\
\hline
\end{tabular}

from clinic, he underwent investigation for PUO in which he had multiple sets of blood cultures collected and 3 sets of isolated lactobacillus (Table 1).

He underwent a whole body scan showing a pool of activity in the right knee suggestive of chronic low-grade persistent infection. A transthoracic echocardiogram and a colonoscopy to investigate the altered bowel habit were carried out and were both negative. At this time a fourth repeat CT abdomen and pelvis was requested. This showed small extramural pockets of gas at the level of the graft bifurcation and a further possible tiny pocket of gas at the cranial aspect of the graft, appearances which would be suspicious for sepsis related to the graft.

At this point, there was multidisciplinary team input from vascular surgeons, vascular radiologists, infectious diseases and microbiology and appropriate management options were discussed. The two available options were (1) explantation of the graft with extra-anatomical bypass and (2) long-term suppressive antibiotics with serial CRP and imaging. In view of his age, that the WCC was within normal limits and the CRP was falling, the equivalence of the CT finding, and the fact that the surgery to remove the graft would carry a significant mortality and morbidity risk, the decision was made to start IV antibiotics for a minimum of 6 wks. The caveat to this approach was that in the event of worsening sepsis despite antibiotics and/or CT evidence of worsening perigraft infection or development of aortoenteric fistula, then surgery would be carried out.

IV Daptomycin $(6 \mathrm{mg} / \mathrm{kg}$ ) (plus gentamicin initially) was commenced after confirmatory MIC testing of the lactobacillus to Daptomycin; this was the most recently and most persistently isolated pathogen. Daptomycin was well tolerated by the patient. At 6 weeks, based on good clinical response, this was changed to oral amoxicillin plus clindamycin, and the patient was discharged home. Within $48 \mathrm{hrs}$ of discharge he represented with rigors and fevers. He was readmitted and a repeat $\mathrm{CT}$ showed increasing air encircling the right most anterior limb of the aortic graft. At this point the decision was made to carry out surgery to remove the graft.
In July 2011, the infected graft was explanted, the space washed out, and bilateral axillofemoral bypass graft inserted. Intraoperatively, a perforated duodenum was reported, which was the likely source of the multiple bacteraemias isolating the variety of gut organisms seen. This was repaired involving a retrocolic gastrojejunostomy and feeding jejunostomy, in an operation lasting more than 10 hours (Figure 1).

To date, the patient remains well at home, the graft remains patent and perfusing the leg, and he has chosen not to continue oral suppressive antibiotics.

\section{Discussion}

Diagnosis of PVGI is extremely challenging. It is based on patient history and examination and parameters such as CRP, WCC, blood cultures (which often remain negative in lateonset graft infection), and 8 radiological imaging, which can be extremely difficult to interpret and in this case was repeatedly negative in the early stages. Unlike early-onset PVGI, which is often attributed to contamination of the graft at or soon after insertion [1] and presents mainly as a postsurgical wound infection, the etiology of late-onset graft infections is far less certain. Presentation can be more occult in manner and many patients present with few or no overt symptoms of disease [1]. There may be local signs of infection such as thrombosis or failure of the graft, false aneurysm, or bleeding (aortoenteric fistula formation). In this case the patient presented with occult signs of graft-duodenal erosion, resulting in the translocation of a variety of gut organisms into the blood stream, likely seeding a potentially osteoarthritic knee joint, which was the initial presenting complaint.

Gold standard treatment involves complex surgery to remove the infected graft. For many patients, however, multiple comorbidities often render this an unfeasible option, leaving long courses of often empirical broad spectrum intravenous antibiotics as the only alternative. A lack of data on the effectiveness of some of the newer antimicrobial agents in prosthetic graft infection limits the treatment options 


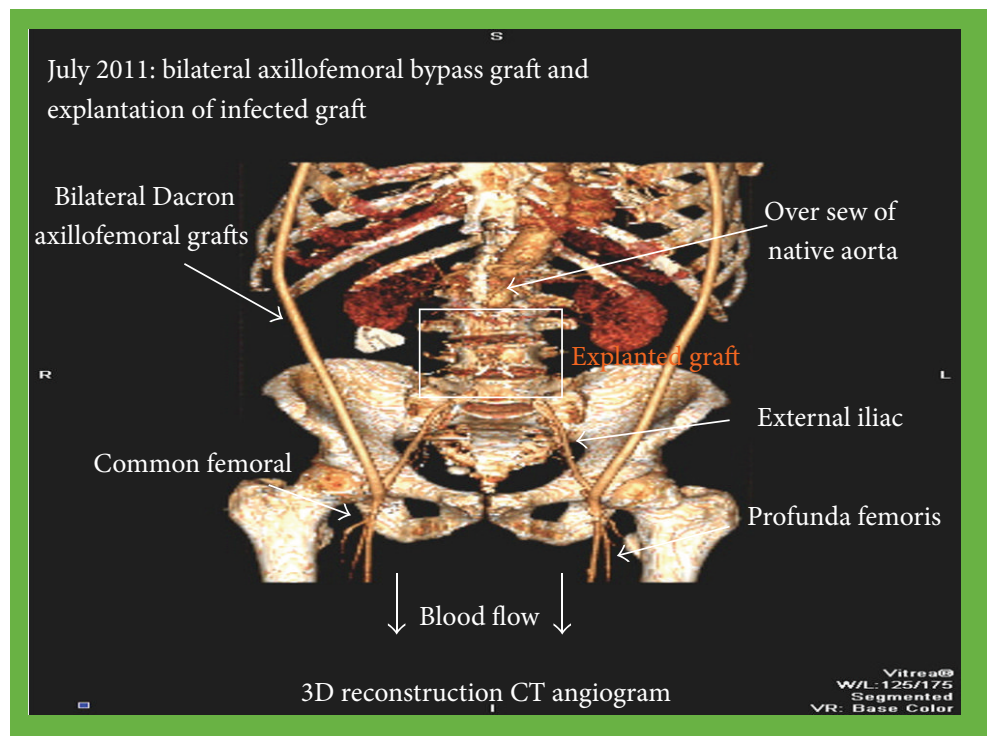

FIGURE 1: 3D-CT angiogram demonstrating removal of the infected aortic graft and extra-anatomical bypass.

available. In this case Daptomycin was used for the suppression of infection. This was an off-licence use of this newer class of antimicrobial agent, and the data to support its use is lacking. Despite the fact that management will often be on a patient-by-patient basis, there is a real and urgent need to develop national consensus treatment guidelines, providing advice on the use of newer antimicrobial agents such as Daptomycin, Linezolid, and Tigecycline in PVGI and on the optimal duration of therapy.

\section{References}

[1] C. E. Edmiston Jr., "Vascular graft acute and late-onset infections," Infectious Diseases in Clinical Practice, vol. 3, no. 2, pp. 147-150, 1994.

[2] V. S. Antonios, A. A. Noel, J. M. Steckelberg et al., "Prosthetic vascular graft infection: a risk factor analysis using a casecontrol study," Journal of Infection, vol. 53, no. 1, pp. 49-55, 2006.

[3] G. Piano, "Infections in lower extremity vascular grafts," Surgical Clinics of North America, vol. 75, no. 4, pp. 799-809, 1995.

[4] G. S. Oderich and J. M. Panneton, "Aortic graft infection: what have we learned during the last decades ?" Acta Chirurgica Belgica, vol. 102, no. 1, pp. 7-13, 2002. 


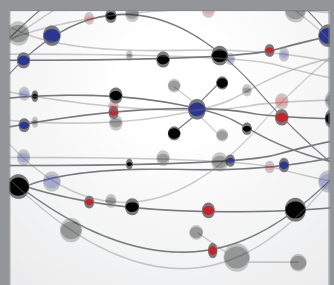

The Scientific World Journal
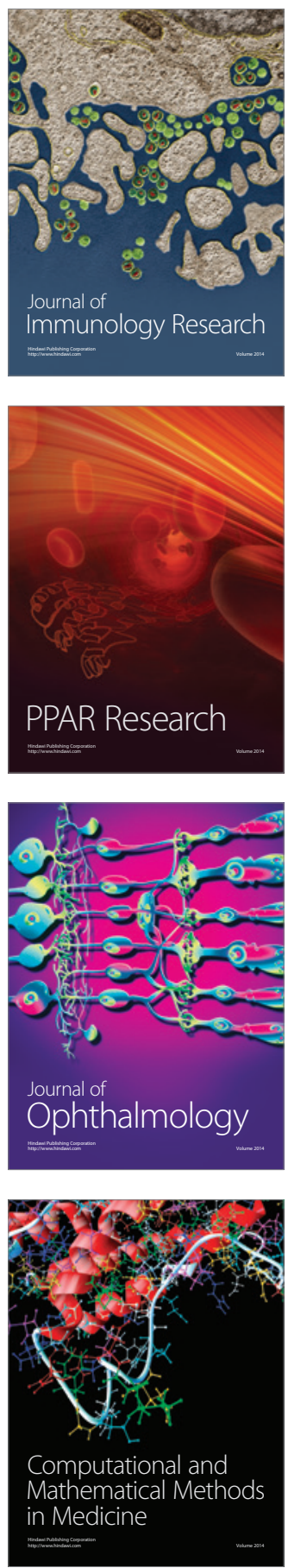

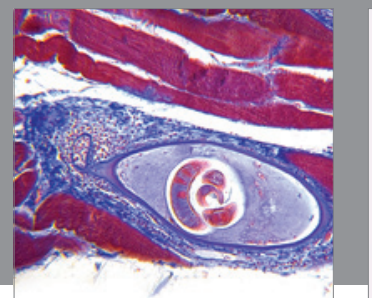

Gastroenterology

Research and Practice
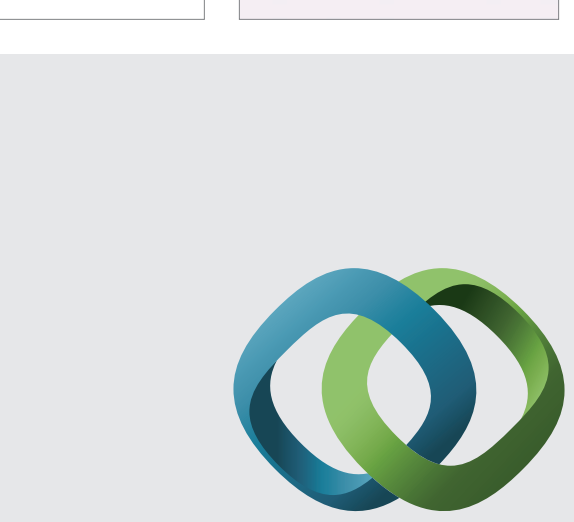

\section{Hindawi}

Submit your manuscripts at

http://www.hindawi.com
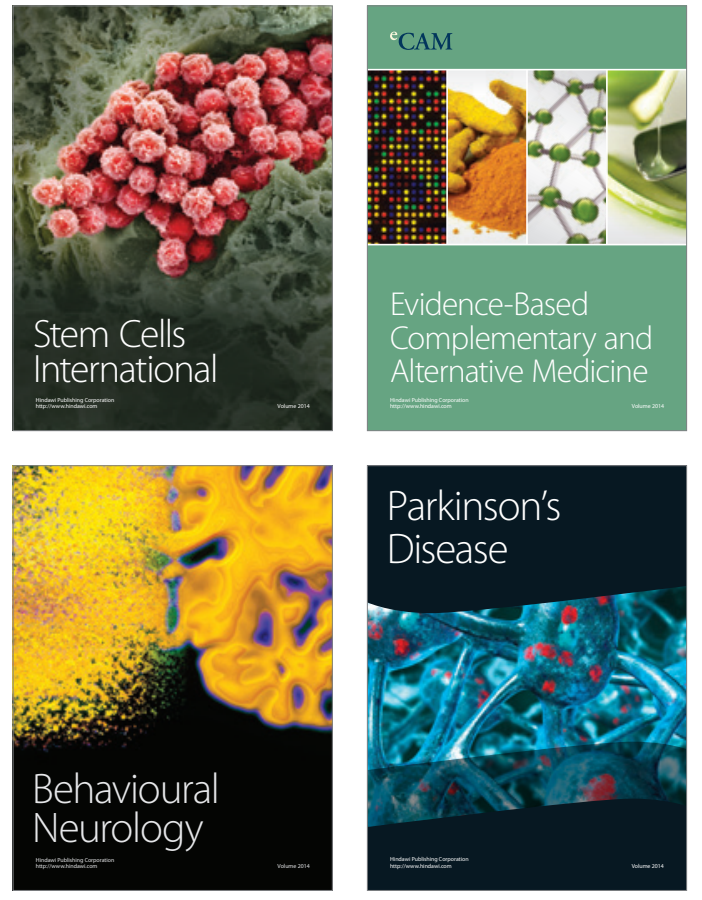
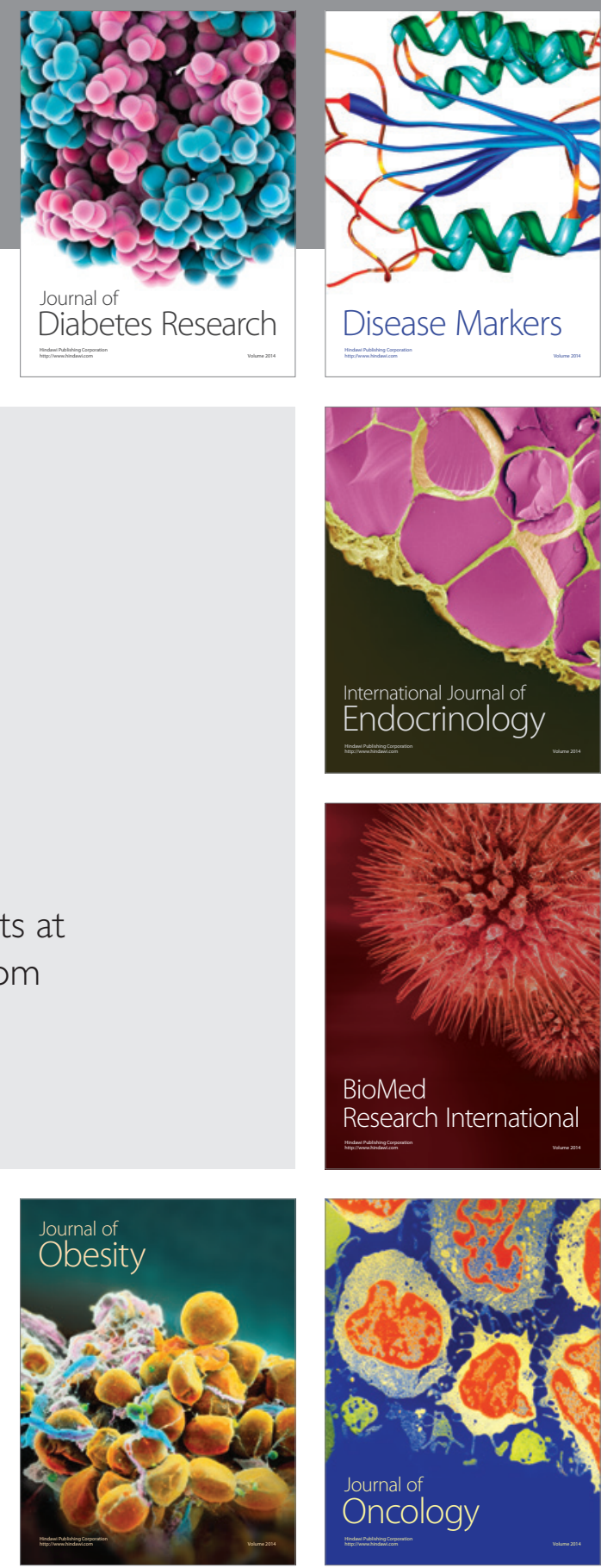

Disease Markers
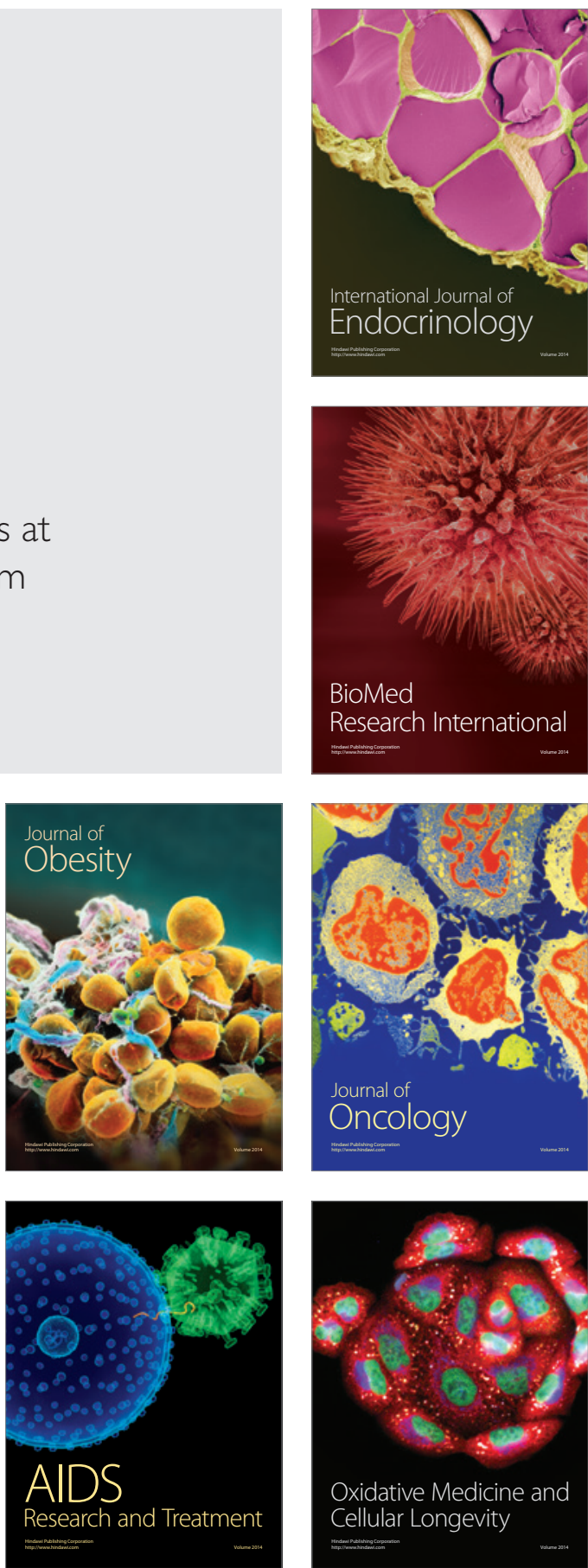